\title{
The effects of water immersion and walking on leg volume, ankle circumference and epifascial thickness in healthy subjects with occupational edema
}

Phlebology

0(0) 1-8

(C) The Author(s) 2021

Article reuse guidelines:

sagepub.com/journals-permissions DOI: I0.I I 77/0268355520984065 journals.sagepub.com/home/phl

(SSAGE

\author{
Giovanni Mosti' (1) and Alberto Caggiati ${ }^{2}$
}

\begin{abstract}
Background: Balneotherapy has been considered beneficial in patients with chronic venous disease due to patientreported positive outcomes on improvement of symptoms and quality of life.

Study aim: Assessing the effects of prolonged water immersion (WI) on leg edema and epifascial thickness and to compare these data with those achieved after continuous walking on ground.

Material and methods: On three consecutive days, 14 otherwise healthy volunteers ( 9 females, 5 males, mean age $53 \pm$ 10 years) affected by occupational edema $(\mathrm{OE})$, defined as the edema developing during the time period of the working day and disappearing overnight, stayed standing immobile in a swimming pool for 30 minutes (30'), continuously walking again for 30' in the same pool and walking on ground for 30' without interruptions in a randomized sequence. Leg volume, ankle circumference and epifascial thickness of both legs were assessed each day before and after each intervention.

Results: Leg volume showed a median reduction by $4.20 \%(I Q R 5-3.6)(P=0.0002)$ after 30 ' of immobile standing immersion and by 6.50\% (IQR 7.30-5.6I) ( $<<0.000 \mathrm{I})$ when the patients walked in the pool. Ankle circumference showed a median reduction by $2.89 \%(I Q R ~ 4.23-2.03)(p=0.02)$ with the subjects staying standing still in water and by 5.98\% (IQR 7.47-4. I4) ( $\mathrm{P}=0.0002)$ after 30' walking in the pool. Epifascial thickness showed a median reduction by 24.35\% (IQR 35.26-22.5) ( $\mathrm{P}<0.000 \mathrm{I}$ ) when the volunteers remained standing still and by 32.66\% (IQR 36.9I-28-84) $(P<0.000 I)$, when walking in water. Leg volumetry showed a median reduction by $0.20 \%(I Q R-0.44-0.29)(P=0.375)$ after walking on ground for 30'. Ankle circumference and epifascial thickness did not show any difference walking on ground compared to baseline situation.

Conclusions: This study showed that 30' of WI, especially when associated with walking, reduced leg volume in otherwise heathy subjects with $O E$ and that walking outside the water did not.
\end{abstract}

\section{Keywords}

Balneotherapy, water immersion, venous rehabilitation, leg volumetry, leg edema, skin thickness

\section{Introduction}

Balneotherapy (physical treatments performed during water immersion) is considered beneficial in patients with chronic venous disease (CVD) based on improvement of symptoms and quality of life reported by patients. ${ }^{1,2}$ A recent Cochrane review highlighted the need for evidence-based data on the effect of balneotherapy in CVD legs. ${ }^{3}$ In their conclusions the Authors recommended to standardize measurements of outcomes such as disease severity score, quality of life, pain, oedema and finally choice of time points during follow-up for future studies. The need to evaluate the objective effects of balneotherapies on vein size, venous hemodynamics and leg volume was not mentioned.

In a study from our group it was demonstrated by underwater Duplex Sonography (uDS), that water

\footnotetext{
'Angiology Department, MD Barbantini Clinic, Lucca, Italy

${ }^{2}$ Anatomy Department, Sapienza University of Rome, Rome, Italy

Corresponding author:

Giovanni Mosti, Clinica MD Barbantini, Via del Calcio n.2, 55I00 Lucca, Italy.

Email: giovanni.mostil0@gmail.com
} 
immersion (WI) elicits an immediate reduction of vein caliber accompanied by the increase of blood return and, in legs with venous insufficiency, the reduction of reflux. ${ }^{4}$ More recently, underwater Strain Gauge Plethysmography (uSGP) showed a decrease of leg volume immediately after the immersion and a further decrease during a short walking into the pool maybe due to an increased ejection fraction (EF) of the calf muscles. ${ }^{5}$

The aim of this study was to evaluate the effects of a prolonged WI on the leg volume and epifascial thickness in otherwise healthy volunteers with pitting occupational edema (OE), defined as the edema developing during the time period of the working day and disappearing overnight. ${ }^{6-9}$ Results obtained after $30^{\prime}$ of immobile standing or continuous walking in a standard pool were compared with those achieved after 30' of continuous walking on ground.

\section{Material and methods}

\section{Study cohort}

Fourteen healthy volunteers ( 9 females, 5 males, mean age $53 \pm 10$ years; BMI range $18-25$ ) working mainly in standing/sitting position (3 nurses, 2 physicians, 7 shop assistants, 2 company employees) showing an evening OE were enrolled in this study and both their legs were tested. Before the test all the volunteers did their usual job that, in our country, lasts eight hours during which all the volunteers worked standing or sitting keeping on for the whole shift.

Inclusion criteria were: age from 18 to 65 years, OE in the absence of venous and lymphatic disorder as assessed by clinical examination, clinical history, and Duplex scanning.

Pregnancy or breast-feeding, venous or lymphatic disorder, absence of $\mathrm{OE}$, presence of systemic diseases or use of drugs possibly causing leg edema were considered exclusion criteria.

\section{Study protocol}

All the volunteers were tested at the same hour in the late afternoon $(6 \mathrm{pm})$ after their working shift performed from the morning to the time test, at the end of Summer 2019 in the Dalia swimming pool (Pietrasanta, Lucca, Italy). Air temperature was $26-28^{\circ} \mathrm{C}$ and the humidity average rate $70 \%$. The volunteers were trained to walk in circle in the pool and to walk along a well-established path on ground with a fixed pace. After the training the volunteers were asked:

- to stay standing still for 30 minutes in the pool (water depth $110 \mathrm{~cm}$, water temperature $30^{\circ} \mathrm{C}$ ) without any standing support.
- to continuously walk, again for $30^{\prime}$, in the same pool at a constant water depth of $110 \mathrm{~cm}$.

- to walk on ground for 30' without interruptions.

The three tests were performed in three consecutive days, in a randomized sequence (list randomizer www. random.org) to minimize all the possible variables. Leg volume, ankle circumference and epifascial thickness measured at ankle level were assessed every day before and after the tests in order to get daily data for every single test. All the measurements were taken by the same doctor in a not blinded setting.

\section{Measuring systems}

Leg volume was measured by water displacement volumetry (WDV). ${ }^{10}$ The right leg was always measured before the left one. The spout of the WDV container was at $38 \mathrm{~cm}$ from the bottom. The water discarded after leg immersion was weighted by an electronic scale.

Ankle circumference was measured by tape $5 \mathrm{~cm}$ above the medial malleolus and an indelible mark was placed in order to repeat measurements in a consistent site. At the same level the thickness of the epifascial layer was measured by Duplex Ultrasound (S9, Sonoscape Shenzen, China), with a 7.5-12 MHz probe according to the "no touch technique": a great amount of gel was used to ensure ultrasound transmission avoiding any contact between the probe and the skin. ${ }^{11}$ Epifascial thickness was measured as the distance between the epidermal echoes and the muscular fascia (so to include both cutaneous and subcutaneous layers) by a line crossing the great saphenous vein lumen to obtain reproducible measurements (Figure 1).

\section{Ethics}

All the volunteers gave their informed consent before enrollment. The study was performed according to the principles of the World Medical Association Declaration of Helsinki. Ethical committee approval is not required by the Italian authorities for observational studies. In addition, all the procedures were noninvasive, without any drug or medical device, of short duration and performed by healthy subjects under continuous medical surveillance.

\section{Statistical analysis}

Median values and interquartile ranges are given as data are not normally distributed. For data comparisons the Friedmann test with multiple comparison was used. Paired Wilcoxon tests were performed to compare two sets of data. The difference between right and left leg and between male and females was not analyzed since the number of subjects was too small 


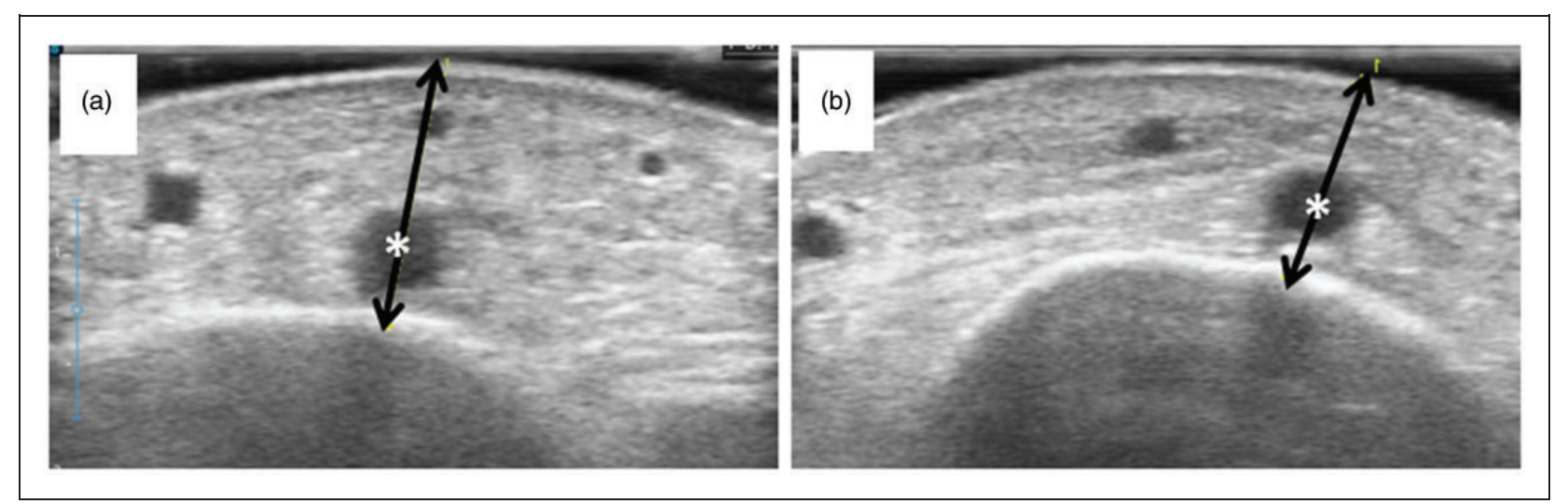

Figure I. Sonographic measurement of skin thickness at the ankle, that was measured by crossing the great saphenous vein $(*)$ lumen. (a) Before immersion (II.86 mm). (b) After 30' standing still into the pool $(10.06 \mathrm{~mm})$. Skin thickness reduction was $19.6 \%$.

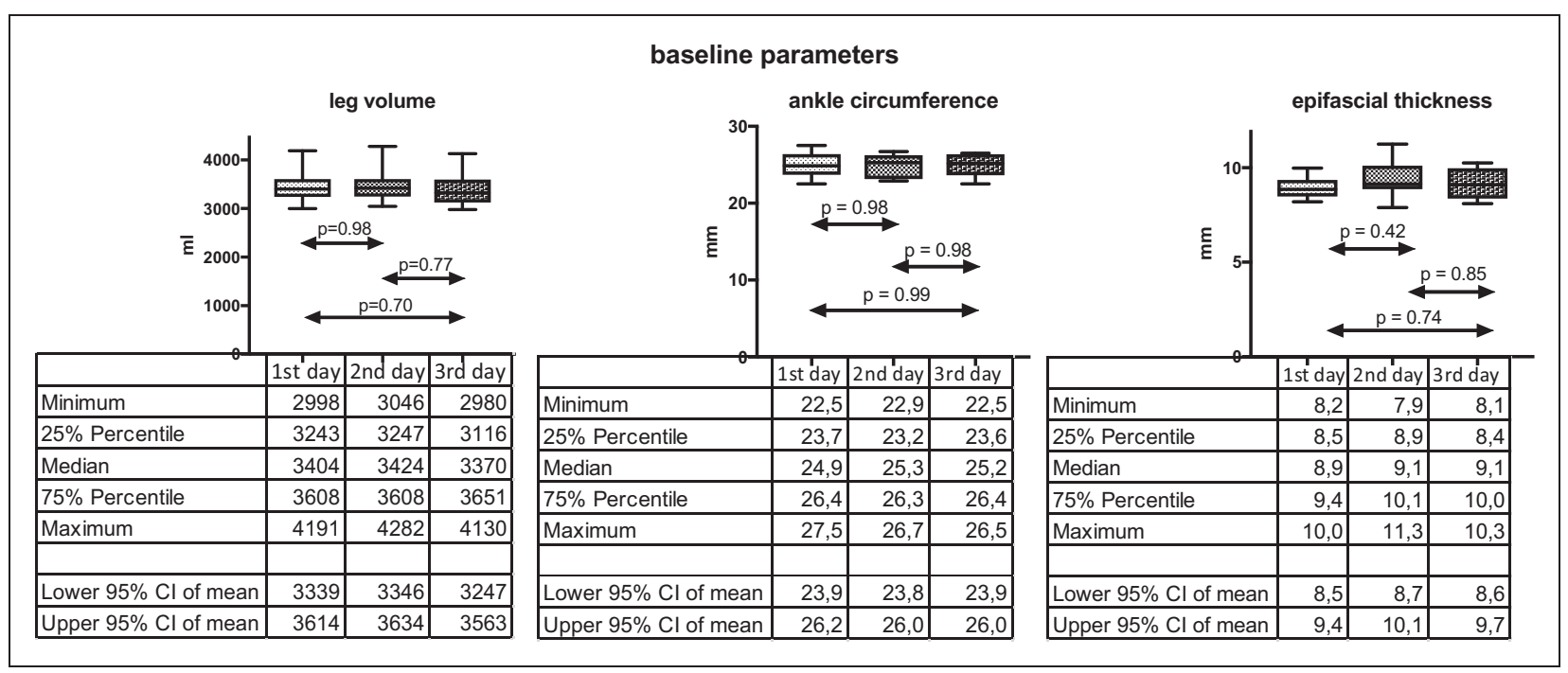

Figure 2. Baseline values of leg volume, ankle circumference and epifascial thickness in the three different days of the tests. Data are reported as medians and interquartile range (IQR).

to make a meaningful comparison. Differences with a $\mathrm{P}<.05$ were considered statistically significant. Statistical evaluations and the graphs were generated by Graph Pad Prism, version 7, software (Graph Pad, San Diego, Ca, USA).

\section{Results}

\section{Baseline measurements}

Leg volume, ankle circumference and epifascial thickness showed only minor, not significant changes in the three consecutive test days (Figure 2).

Leg volume. Compared to the baseline value of $3404 \mathrm{ml}$ (IQR 3243-3608 ml), the leg volume significantly decreased to $3255 \mathrm{ml}$ (IQR $3102-3467 \mathrm{ml}$ ), with a median difference by $149 \mathrm{ml}(-4.20 \%)$ after 30 minutes of immobile standing immersion $(p=0.0002)$. Leg volume decreased even more from $3424 \mathrm{ml}$ (IQR $3247-3608 \mathrm{ml}$ ) to $3188 \mathrm{ml}$ (IQR $3027-3364 \mathrm{ml}$ ) with a median difference by $236 \mathrm{ml}(-6.50 \%)$, when the patients walked in the pool $(\mathrm{p}<0.0001)$. The difference between leg volume reduction after immobile immersion and after walking in water was statistically significant ( $\mathrm{p}<0.0001)$. After walking on ground for $30^{\prime}$ leg volumetry showed a very small and not significant reduction from $3370 \mathrm{ml}$ (IQR $3116-3651 \mathrm{ml}$ ) to $3355 \mathrm{ml}$ (IQR 3125-3646 ml) with a difference by $15 \mathrm{ml}(-0.20 \%)$ after walking (Figure 3).

Ankle circumference. Compared to baseline value of $24.85 \mathrm{~cm}$ (IQR $23.68-26.38 \mathrm{~cm}$ ), the ankle circumference significantly decreased to $24.10 \mathrm{~cm}$ (IQR 22.9-25.85), 


\begin{tabular}{|l|c|r|r|r|r|r|}
\hline \multicolumn{1}{|c|}{ LEG VOLUMETRY } & (c) \\
\hline
\end{tabular}

Figure 3. Leg volumetry before and after: (a) standing in water, (b) walking in water, (c) walking on ground. Data are reported as medians and interquartile range (IQR).

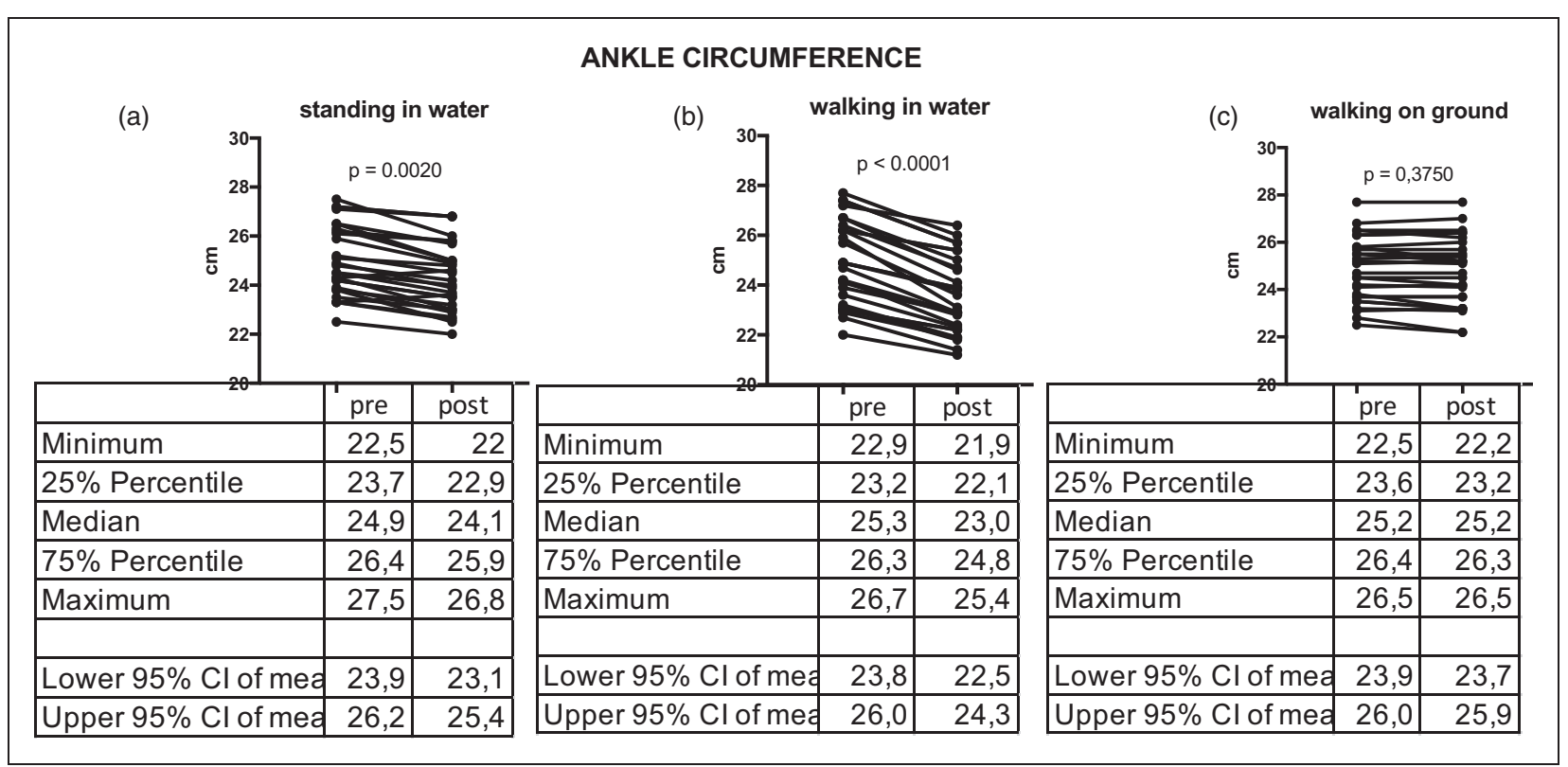

Figure 4. Ankle circumference before and after: (a) standing in water, (b) walking in water, (c) walking on ground. Data are reported as medians and interquartile range (IQR).

with a median difference by $0.75 \mathrm{~cm}(-2.89 \%)$ with the subjects staying standing still in water $(\mathrm{p}=0.02)$. Ankle circumference decreased even more after $30^{\prime}$ ' walking in the pool from $25.30 \mathrm{~cm}$ (IQR $23.15-26.26 \mathrm{~cm}$ ) to $23 \mathrm{~cm}$ (IQR $22.13-24.78 \mathrm{~cm}$ ) with a median difference by $2.3 \mathrm{~cm}(-5.98 \%)(\mathrm{p}=0.0002)$. Again, the reduction of ankle circumference after walking was significantly greater than that after standing still $(p=0.003)$. Walking for $30^{\prime}$ on ground did not change the ankle circumference that measured $25.2 \mathrm{~cm}$ (IQR 23.63-26.35) before walking and $25.2 \mathrm{~cm}$ (IQR 23.2-26.25) after walking (Figure 4). 
Epifascial thickness. Compared to the baseline value out of the water of $8.86 \mathrm{~mm}$ (IQR $8.47-9.36 \mathrm{~cm}$ ), the epifascial thickness decreased to $6.7 \mathrm{~mm}$ (IQR $6.16-6.85 \mathrm{~mm}$ ) with a difference by $2.16 \mathrm{~mm}(-24.35 \%)(\mathrm{p}<0.01)$ when the volunteers remained standing still and from $9.13 \mathrm{~cm}$ (IQR 8.87-10.2 mm) to 6.68 (IQR 6.13-7.28) with a difference by $2.45 \mathrm{~mm}(-32.66 \%)(\mathrm{p}<0.0003)$ after walking. The reduction of epifascial thickness after walking was significantly greater than that after immobile standing $(p=0.009)$. Walking on ground for $30^{\prime}$ did not produce a significant variation of epifascial thickness that remained basically unchanged: $\mathrm{mm} 9.09$ (IQR 8.37$9.97 \mathrm{~mm}$ ) and $\mathrm{mm} 9.05$ (IQR $8.25-9.22 \mathrm{~mm}$ ) pre- and post-walking on ground respectively (Figure 5).

Minor differences between baseline measurements are due the fact that leg volume, epifascial thickness and ankle circumference were evaluated in the evening of the three different days.

\section{Discussion}

Objective hemodynamic and morphologic parameters regarding beneficial effects of balneotherapy in patients with CVD, were poorly evaluated in the past ${ }^{12-14}$ and most of these studies are not fully accepted because of pitfalls in methodology. ${ }^{3}$ Very few studies have objectively demonstrated the effects of the immersion into a pool on the venous system. Only recently were the immediate effects of WI on the leg veins and calf volume investigated. ${ }^{4,5}$

In this study we assessed the effects of prolonged WI on $\mathrm{OE}$ that consists in leg edema occurring in the evening due to daytime activities (even in the absence of venous disease) $)^{6-9}$ and is "the result of a physiologic phenomenon. It is caused by extravasation of fluid from the venules because of a steadily increased venous pressure in the dependent regions of the body, owing to gravity". ${ }^{6}$

OE disappears overnight to reappear at the end of the following working day as proved by the baseline assessment of the three examined parameters showing a minor daily variability without any statistically significant difference (Figure 2). This is why we could perform the three tests on three consecutive days without waiting for a longer time as already shown in previous studies on occupational edema. ${ }^{6}$ In addition, the consistency of the daily baseline value of the three parameters excluded the possible influence of previous tests on the subsequent result. Regardless, in order to avoid any possible training effect and to minimize the possible variables, the sequence of the tests was randomized.

In our study we demonstrated that WI is effective in reducing OE both by standing still or by continuously walking.

Immobile standing for $30^{\prime}$ in a $110 \mathrm{~cm}$ deep pool resulted in a significant reduction of the leg volume, ankle circumference and epifascial thickness. Leg volume reduction occurring after static immersion is due to the leg compression by water Hydrostatic Pressure (wHP). According to the Stevin's law, ${ }^{15}$ a $110 \mathrm{~cm}$ column of water exerts, at bottom of the pool, a wHP of $81 \mathrm{mmHg}$ decreasing by $0.73 \mathrm{mmHg}$ per centimeter upwards. Therefore, we can assume that in standing position, wHP ranges approximately between

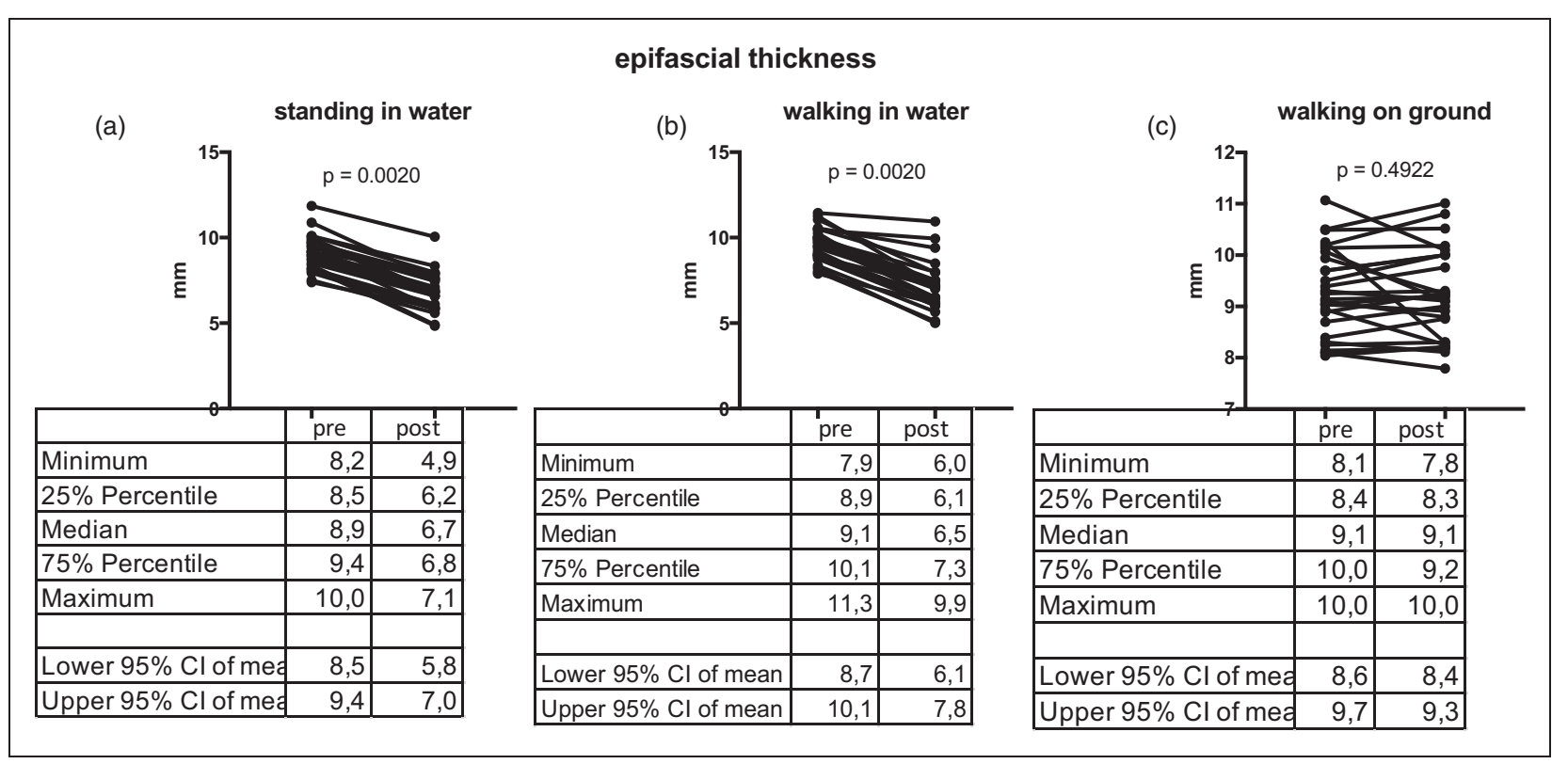

Figure 5. Epifascial thickness before and after: (a) standing, (b) walking in water, (c) walking on ground. Data are reported as medians and interquartile range (IQR). 
65-70 $\mathrm{mm} \mathrm{Hg}$ at gaiter level and between 55-60 mm Hg at the mid-calf. This strong pressure could explain the great leg volume reduction in our case series that is significantly higher ${ }^{6,16}$ or similar ${ }^{17,18}$ compared to other studies evaluating the effects of graduated compression stockings. Unfortunately, in these other studies $^{16-18}$ different protocols and measuring systems make detailed comparison with our results challenging.

The reduction of the calf volume occurring immediately after immersion shown by $\mathrm{uSGP}^{5}$ is related to the reduction of intravascular volume by narrowing the deep and superficial veins, as demonstrated by $\mathrm{uDS}^{4}$ Prolonging the immersion, the wHP causes a further leg volume decrease mainly due to the reduction of the epifascial layer thickness. The reduction of the fluid content of the epifascial layer may be explained by the reduction of venular fluid filtration and by the improvement of the peripheral lymphatic drainage due to wHP. The squeezing effect of wHP on leg tissue most likely forces the interstitial fluid into the lymphatic vessels as venous reabsorption has been excluded by the new knowledge on tissue fluid exchange. ${ }^{19}$ On the contrary, it has been shown that compression increases the tension on the anchoring filaments of the initial lymphatics by increasing the tissue pressure. Initial lymphatics will be opened and lymphatic flow will be pushed towards the lymph collectors by their valves. Rhythmic autonomous contractions of the collectors provide the main force of further lymph drainage. ${ }^{20}$ Also leg bioimpedance demonstrated that the reduction in volume of the leg is due to a reduction of the extracellular water both after walking with compression garments, ${ }^{17}$ or after great saphenous vein catheter foam. ${ }^{21}$

Comparing in-water immobile standing and exercising, the latter resulted in a significantly greater reduction of the leg volume ( $236 \mathrm{ml} \mathrm{vs} 150 \mathrm{ml}$ as an average), ankle circumference and epifascial thickness. A mutual enhancement of the effects of wHP and muscle pumping on venous hemodynamics and lymphatic reabsorption may explain these outcomes. It was actually shown that muscle contraction acts as an extrinsic pump on lymphatic flow so increasing interstitial fluid reabsorption. ${ }^{22,23}$

WDV did not show any significant reduction of the leg volume and ankle circumference in the same subjects after walking on ground for 30' at precisely the same time of the day and in the same atmospheric conditions. Even if this observation is in conflict with the common belief that walking facilitates peripheral drainage, it was already described by other authors by using different measuring systems: impedance plethysmography, ${ }^{24}$ strain gauge plethysmography, ${ }^{25}$ and "truncated cone formula". ${ }^{17}$ These studies could be theoretically criticized because strain gauge plethysmography measures only segmental volume variations of the calf, whereas the "truncated cone measurement" excludes the foot volume. However, our data obtained by WDV, that is considered the gold standard for leg volume measurement, ${ }^{10}$ confirm that leg volume does not decrease by walking in patients with OE. Volume reduction only occurs when walking is associated with stockings wearing. ${ }^{8,17,26}$

Our findings are in agreement with previous studies reporting a WI-related reduction of leg edema. ${ }^{27-30} \mathrm{In}$ all these studies the volume reduction was demonstrated utilizing more complex protocols which sometimes $^{28,30}$ required longer WI time, and multiple exercises that required assistant personnel for some patients, especially the older ones. Furthermore, in one of these studies, the effects of WI were evaluated in pregnant women in whom the edema is related to fluid and salt retention in addition to dependency and activity restrictions. In a more recent study in patients with chronic leg edema, the effects of a long series of exercises performed in a rehabilitation pool were not compared with those of similar activities when performed on-ground. ${ }^{30}$

It is noteworthy to consider that, despite of more complex protocols, it is our impression that it can be challenging to standardize walking, to check the correct execution of bodyweight exercises, to assess the reproducibility of the exercises, and to objectively quantify the real extent of underwater muscular activities.

Our protocol, based on simple walking in water, does not require a rehabilitative pool nor the assistance of dedicated personnel and can be feasible independently by everybody, even by patients with neuromuscular or osteoarticular impairment.

Finally, it is important to observe that our results were achieved with water height of $110 \mathrm{~cm}$ to exert a strong pressure on the leg tissues. Possible differences related to patient height are negligible because, in these conditions, the wHP at the bottom of the pool is higher than $70 \mathrm{~mm} \mathrm{Hg}$ and able to counteract the intravenous pressure in the standard population.

One weakness of this study regards the epifascial thickness measurement. In practice, measuring exactly the epifascial thickness at precisely the same point of the leg and at the same angle can be sometimes difficult. It is true that the reduction of epifascial thickness was consistent in all the measurements and systematically correlates with leg volume reduction. Another weakness is that we have not enrolled patients with CVD or lymphedema, but just included healthy volunteers with OE. However, OE is due to venous stasis and lymphatic impairment and is treated by compression stockings like CVD. ${ }^{6,31}$ Accordingly, we believe that OE represents an optimal model for a basic study on the effects of WI in CVD patients in which edema can 
be related to different pathophysiologic mechanisms (obstruction, incompetence, inflammation, etc). Further and larger studies are necessary to draw conclusions about the amount and duration of clinical effects of a complete balneotherapy treatment and to compare the effect of balneotherapy with other modalities of mechanical treatment of leg edema.

\section{Conclusions}

This study proved that a prolonged WI is effective in reducing leg edema and that underwater walking enhances this phenomenon. This is possibly due to the combined effects of wHP and muscle pumping on venous hemodynamics and lymphatic drainage. An in-progress study includes that patients with CVD will evaluate the possible implication of underwater walking in the treatment and rehabilitation of venous diseases. ${ }^{32}$

\section{Declaration of Conflicting Interests}

The author(s) declared no potential conflicts of interest with respect to the research, authorship, and/or publication of this article.

\section{Funding}

The author(s) received no financial support for the research, authorship, and/or publication of this article.

\section{Ethical approval}

Not requested in Italy for observational studies not involving drugs od devices as in our study.

\section{Guarantor}

GM.

\section{Contributorship}

Both authors contributed in preparing the protocol, performing the study and writing the manuscript. GM was involved in performing the statistical analysis.

\section{ORCID iD}

Giovanni Mosti (D) https://orcid.org/0000-0001-7639-8463

\section{References}

1. Carpentier PH, Blaise S, Satger B, et al. A multicenter randomized controlled trial evaluating balneotherapy in patients with advanced chronic venous insufficiency. J Vasc Surg 2014; 59: 447-454.

2. Forestier RJ, Briancon G, Francon A, et al. Balneohydro-therapy in the treatment of chronic venous insufficiency. Vasa 2014; 43: 365-371.

3. de Moraes Silva MA, Nakano LC, Cisneros LL, et al. Balneotherapy for chronic venous insufficiency. Cochrane Database Syst Rev 2019; 8: CD013085.
4. Caggiati A, Lattimer C, Kalodiki E, et al.; Underwater Compression Group. Underwater sonography of leg veins. EJVES Short Rep 2018; 41: 13-15.

5. Mosti G, Bergamo G, Oberto S, et al. The feasibility of underwater computerized strain gauge plethysmography and the effects of hydrostatic pressure on the leg venous hemodynamics. EJVES Vasc Forum 2020; 47: 60-62.

6. Partsch H, Winiger $\mathbf{J}$ and Lun B. Compression stockings reduce occupational leg swelling. Dermatol Surg 2004; 30 : 737-743.

7. Mosti $G$ and Partsch H. Occupational leg oedema is more reduced by antigraduated than by graduated stockings. Eur J Vasc Endovasc Surg 2013 May; 45: 523-527.

8. Quilici Belczak CE, Pereira de Godoy JM, Seidel AC, et al. Comparison of $15-20 \mathrm{mmHg}$ versus $20-30 \mathrm{mmHg}$ compression stockings in reducing occupational oedema in standing and seated healthy individuals. Int $J$ Vasc Med 2018; 2018: 2053985.

9. Shibasaki S, Kishino T, Yokoyama T, et al. Sonographic detection of physiological lower leg oedema in the late afternoon in healthy young women. Clin Physiol Funct Imaging 2020; 40: 381-384.

10. Rabe E, Carpentier P and Maggioli A. Understanding lower leg volume measurements used in clinical studies focused on venous leg edema. Int Angiol 2018; 37: 437-443.

11. Caggiati A. Ultrasonography of skin changes in legs with chronic venous disease. Eur J Vasc Endovasc Surg 2016; 52: 534-554.

12. Ernst E, Saradeth T and Resch KL. A single blind randomized, controlled trial of hydrotherapy for varicose veins. Vasa 1991; 20: 147-155.

13. Mancini S Jr, Piccinetti A, Nappi G, et al. Clinical, functional and quality of life changes after balneokinesis with sulphurous water in patients with varicose veins. Vasa 2003; 32: 26-30.

14. Hartmann B, Drews B, Kayser T, et al. Venous function in patients with venous disease and healthy controls before and after a bathing procedure and subsequent cold stimulus. J Japanese Assoc Phys Med Balneol Climatol 1995; 58: 134-134.

15. Bettini A. A course in classical phisics-2. Fluids and thermodynamics. Berlin: Springer p.8.

16. Wou J, Williams KJ and Davies AH. Compression stockings versus neuromuscular electrical stimulation devices in the management of occupational leg swelling. Int $J$ Angiol 2015; 25: 104-109.

17. Gianesini S, Raffetto JD, Mosti G, et al. Volume control of the lower limb with graduated compression during different muscle pump activation conditions and the relation to limb circumference variation. J Vasc Surg Venous Lymphat Disord 2020; 8: 814-820.

18. Gianesini S, Mosti G, Sibilla MG, et al. Lower limb volume in healthy individuals after walking with compression stockings. J Vasc Surg Venous Lymphat Disord 2019; 7: 557-561.

19. Levick JR and Michel CC. Microvascular fluid exchange and the revised starling principle. Cardiovasc Res 2010; 87: $198-210$. 
20. Moffatt C, Partsch H, Schuren J, et al. International lymphoedema framework position document. pp.1516. Deborah Glover, Editor. Imprimerie Reboul, SaintÉtienne, France. June 2012

21. Cavezzi A, Mosti G, Colucci R, et al. Compression with $23 \mathrm{mmHg}$ or $35 \mathrm{mmHg}$ stockings after saphenous catheterfoam sclerotherapy and phlebectomy of varicose veins: a randomized controlled study. Phlebology 2019; 34 : 98-106.

22. Moore JE Jr and Bertram CD. Lymphatic system flows. Annu Rev Fluid Mech 2018; 50: 459-482.

23. Olszewski W. Contractility patterns of human leg lymphatics in various stages of obstructive lymphedema. Ann N Y Acad Sci 2008; 1131: 110-118.

24. Stick C, Stöfen P and Witzleb E. On physiological edema in man's lower extremity. Eur J Appl Physiol Occup Physiol 1985; 54: 442-449.

25. Stick C, Hiedl U and Witzleb E. Volume changes in the lower leg during quiet standing and cycling exercise at different ambient temperatures. Eur J Appl Physiol Occup Physiol 1993; 66: 427-433.

26. Quilici Belczak CE, Pereira De Godoy JM, Quilici Belzack S, et al. Compression stockings have a synergistic effect with walking in the late afternoon to reduce edema of the lower limbs. Int Angiol 2012; 31: 490-493.

27. Irion JM and Irion GL. Water immersion to reduce peripheral edema in pregnancy. $J$ Women's Health Phys Ther 2011; 35: 46-49.

28. Hartmann S and Huch R. Response of pregnancy leg edema to a single immersion exercise session. Acta Obstet Gynecol Scand 2005; 84: 1150-1153.

29. Kent T, Gregor J, Deardorff L, et al. Edema of pregnancy: a comparison of water aerobics and static immersion. Obstet Gynecol 1999; 94: 726-729.

30. Gianesini S, Tessari M, Bacciglieri P, et al. A specifically designed aquatic exercise protocol to reduce chronic lower limb edema. Phlebology 2017; 32: 594-600.

31. Amsler F and Blattler W. Compression therapy for occupational leg symptoms and chronic venous disorders e: a meta-analysis of randomised controlled trials. Eur J Vasc Endovasc Surg 2008; 35: 366-372.

32. Caggiati A, De Maeseneer M, Cavezzi A, et al. Rehabilitation of patients with venous diseases of the lower limbs: State of the art. Phlebology 2018; 33: 663-671. 